\title{
Naturaleza del deseo intelectivo en Aristóteles
}

The nature of intellective desire in Aristotle's thought

\author{
Magdalena Bosch Rabell ${ }^{1}$ \\ Universidad Internacional de Catalunya
}

Recibido: 02.08.2021

Aceptado: 30.09.2021

\section{Resumen}

Este artículo analiza y reivindica el concepto de deseo intelectivo en Aristóteles. Este es un concepto que acoge las formas de deseo vinculadas a la razón y por ello pertenecientes al alma teórica (theoretike psyqué). Es un tema llamativamente descuidado desde que Kant determina la legitimidad exclusiva de la razón en la moralidad. En época poskantiana resulta realmente difícil reconocer el protagonismo originario del deseo en la obra Aristotélica, pues por la tradición kantiana en que estamos inmersos, nuestra lectura a lo largo del siglo XX se centró en la razón y en sus imperativos. La Ética Nicomáquea permite una nueva comprensión del deseo intelectivo si se reconoce su prioridad. Una ética eudemonista es, necesariamente, una ética del deseo; y la felicidad es su plenitud. Para llevar a cabo este análisis tratamos en, primer lugar, la discusión existente sobre el intelectualismo aristotélico. A continuación, se exponen elementos que muestran la importancia del deseo en la Ética Aristotélica. En la tercera sección se argumenta cómo se ha asumido generalmente un falso conflicto entre razón y deseo. En la siguiente, se analiza la relación de boulesis y orexis y al final se ponen en relación ambas con la proareis señalando que también es un modo de deseo.

\footnotetext{
${ }^{1}$ mbosch@uic.es

Volumen 2. Número 1. Enero - Junio 2022

ISSN: 2745-0333 (En línea)
}

https://orcid.org/0000-0002-9292-6964 
Palabras clave: Aristóteles, deseo, voluntad, elección, proairesis.

\begin{abstract}
This article analyses and reclaims the concept of intellective desire in Aristotle's work. It is a concept that includes the different kinds of desire linked to reason and, therefore, belonging to the "theoretical soul" (theoretike psyqué). This is a neglected topic since Kant determined the exclusive legitimacy of reason for morality. In poskantian times is really hard to recognize the original prominence of desire in Aristotle's thought, since the Kantian tradition in which we are immerse, our reading along the XX century was focussed on the reason and its imperatives. The Nicomachean Ethics allows a new comprehension of the intellective desire when we assume its priority. A eudemonist ethics is, necessarily, an Ethics of desire and happiness is its height. To elaborate this analysis we explore, at the beginning, the existent discussion about the Aristotelian intellectualism. Secondly, the key elements that show the importance of desire in Aristotelian Ethics are exposed. Third, we argue that the general assumption about a conflict between reason and desire in the Aristotelian thought is false: The true conflict takes place between two different kind of desire. The fifth section explains the meaning of willing (boulesis) as a desire (orexis) of the good and, finally, choice (proairesis) is studied in its desiring dimension.
\end{abstract}

Keywords: Aristotle, desire, willing, choice, proairesis

\title{
Introducción
}

En 1975 Kraut revisó el papel del amor en la ética Aristotélica. La consideró, como conclusión de su estudio, una ética del amor. Para Kraut la importancia del amor se descubre desde la comprensión de las virtudes: las virtudes están relacionadas con el amor y la felicidad. Esta propuesta marcaba un hito en la superación del intelectualismo aristotélico. 
La hipótesis principal de Kraut se podría resumir en el siguiente enunciado: La ética de Aristóteles es una ética de la felicidad y la felicidad incluye el amor. Por eso se la califica de inclusiva; porque incluye el amor y otros elementos relacionados con las virtudes, además de la vida intelectiva que todos consideran el contenido esencial de la felicidad aristotélica (Kraut, 1975). Las discusiones posteriores se centraron en esta noción de felicidad inclusiva: su contenido, su significado y si realmente superaba el intelectualismo.

A lo largo de estas discusiones el amor que Kraut intentaba rescatar como cuestión central de la ética aristotélica, se entendió solo como un complemento de la felicidad, para afirmar que la felicidad es inclusiva y no se reduce a conocimiento. Buenos ejemplos de ello son los artículos de Nagel (1980) y Akrill (1980) sobre la felicidad. Pero los textos aristotélicos conceden, en efecto, un protagonismo al amor que va más allá de un matiz sobre la felicidad y que no se ha reconocido aún por completo.

La centralidad del deseo sigue pasando inadvertida por el modo en que hemos leído a Aristóteles a lo largo del siglo veinte. La lectura de la ética aristotélica desde finales de siglo XVIII y principio del XIX está impregnada de un esquema antropológico kantiano, pero no aristotélico. Este esquema se concreta especialmente en la idea de que lo único racional es la razón y los deseos son siempre ajenos a ella. Ser más críticos con nuestra propia herencia cultural, nos haría capaces de ver que quizás hemos leído a Aristóteles con esquemas filosóficos propios de nuestros precedentes más próximos. El intelectualismo aristotélico quizás es más un prejuicio de la antropología moderna que un rasgo de la ética del estagirita.

Este artículo propone una lectura de Aristóteles basada en el contexto de sus propias obras capitales y pone entre paréntesis la lectura mediatizada por la mentalidad kantiana. Si nos libramos del prejuicio racionalista, podemos descubrir en los textos del estagirita un concepto que permite superar el intelectualismo: el deseo intelectivo. Se trata de un deseo vinculado al nous, que forma parte esencial de la vida contemplativa, que pertenece al alma teórica y que juega un papel decisivo en el comportamiento virtuoso. La existencia de este deseo intelectivo 
desmiente el intelectualismo de Aristóteles porque muestra que el alma teórica -la parte más esencial del ser humano- incluye deseo: no es solo razón, ni solo conocimiento.

Esta introducción sobre los antecedentes del problema nos lleva a una conclusión clara: la necesidad de revisar la comprensión del deseo intelectivo en Aristóteles. Al comprender su naturaleza, se puede redimensionar su protagonismo y la correspondiente equiparación con la importancia que se concede al nous.

\section{Importancia del deseo en la ética aristotélica}

Para comprender la importancia del deseo en la obra de Aristóteles, debemos considerar primero el marco teleológico de toda su Filosofía. Es muy destacable esta fundamentación en diversas disciplinas y obras: en la Física (el orden de la naturaleza a un fin), la Metafísica (el concepto de causa final), la Ética (la finalidad de la acción humana) o la Retórica (su justificación como arte por la finalidad de persuadir).

Si queremos focalizarnos en la acción humana, resulta especialmente idóneo el inicio de la Ética Nicomaquea: “Toda arte y toda investigación, y del mismo modo toda acción y elección, parecen tender (efíestai) a algún bien; por eso se ha dicho con razón que el bien es aquello a que todas las cosas tienden (efíetai)" (Aristóteles, 1999, 1094 a).

Esta tendencia tiene su paralelo en la Física, en el orden de la naturaleza: también la acción humana tiene una tendencia, una inclinación. Pero el ser humano puede pensar sus tendencias, deliberar y elegir sobre ellas. Por eso la educación del deseo se convierte en un arte: luchar con el deseo es lo más difícil y "de lo más difícil se hace un arte":

Pero además es más difícil luchar con el placer que con la ira, como dice Heráclito, y lo más difícil es siempre objeto del arte y de la virtud, pues hasta lo bueno es mejor en este caso. De suerte que también por esta razón toda la atención, tanto de la virtud 
como de la política, versa sobre el dolor y el placer, puesto que el que se sirve bien de ellos será bueno, y el que lo hace mal, malo. (Aristóteles, 1999, 1105 a 10)

Las tendencias hacen fácil o difícil las acciones. Por eso la educación moral consiste en educar las tendencias, entre las que destaca el deseo, pues ellas pueden hacer fácil la acción Buena.

Si el deseo se orienta a una finalidad que mueve nuestra acción, es importante el contenido de ese deseo: es importante cada acción, su fin, y el fin último al que se dirige toda actividad. En el segundo capítulo del primer libro de la EN se ofrece un argumento de la necesidad de un fin último para que el deseo no sea "vano": “...y no elegimos todo por otra cosa -pues así se seguiría hasta el infinito, de suerte que el deseo sería vacío y vano" (Aristóteles, 1999, 1094 a 20).

Como punto central en el análisis ético aparece el deseo, porque es decisivo en el comportamiento. Lo que mueve la acción siempre es un deseo: hasta el punto de que no hay acción sin él. No basta el conocimiento para mover a actuar. Aristóteles lo dice expresamente en el De Anima: "la observación muestra que el intelecto no mueve sin deseo: la boúlesis (deseo racional) es, desde luego, un tipo de deseo (órexis), y, cuando uno se mueve en virtud de un razonamiento, es que se mueve en virtud de una boúlesis. (...) lo que causa el movimiento es siempre el objeto deseable, que, a su vez, es lo bueno o lo que se presenta como bueno. Pero no cualquier objeto bueno, sino el bien realizable a través de la acción. (...) Es, pues, evidente que la potencia motriz del alma es lo que se llama deseo (órexis)", (Aristóteles, 2020, 433a23-30).

El deseo es además un indicador del bien y de la perfección, porque hay una correspondencia entre los modos de desear y los modos de bien: "Llamamos más perfecto al bien que se persigue (aireton) por sí mismo que al que se busca por otra cosa y al que nunca se elige por otra cosa". (Aristóteles, 1999, 1097 a 30) El que nunca se elige por otra cosa es el fin último, más allá del cual ya nada se puede desear, porque él responde por completo a todas las aspiraciones posibles. Los deseos están subordinados unos a otros y solo el fin último puede 
ocupar el lugar último de esta cadena de subordinaciones. Esto solo es posible si ese fin es realmente "lo Bueno y lo mejor" (Aristóteles, 1999, 1094 a 23) y ha de ser el "bien del hombre" (Aristóteles, 1999, 1094 b 5). Es decir, Bueno para el ser humano en cuanto ser humano, globalmente; no solamente Bueno para un aspecto concreto de su vida.

Aristóteles concede gran importancia a las emociones dentro de la educación moral: “complacerse (hedoné) y dolerse (lupo) como es debido, en esto consiste la buena educación". (Aristóteles, 1999, 1104b 5). Luego la educación moral procura que las pasiones (placer y dolor), y con ellas los diversos modos de deseo que les son propias, estén en coherencia con los deseos racionales: la voluntad (boulesis) y la elección (proairesis). Los deseos racionales, por su vínculo con la razón, “entienden” o "ven” lo que es verdaderamente bueno, por tanto, se orientan al bien de modo casi indefectible.

\section{El falso conflicto entre razón y deseo}

Nadie discute que la ética Aristotélica es una Ética de la felicidad. Sin embargo, sí es necesario revisar cómo ha de entenderse la felicidad. Algunos textos aristotélicos apoyan la comprensión intelectualista de la felicidad. Hay pasajes de la EN que realmente sugieren que la razón es la única facultad que tiende al bien, en contra de la tendencia del deseo. Pondré dos ejemplos:

El primero, sugiere el enfrentamiento entre razón y deseo en estos términos:

...la opinión universal que nos prohíbe gustar frente al placer del gusto: "la primera nos dice que lo rehuyamos, pero el deseo nos mueve a ello, porque puede mover todas las partes; de suerte que ocurre que somos incontinentes movidos en cierto modo por la razón y la opinión, opinión que no se opone a la recta razón por sí misma. (Aristóteles, 1999, 1147 a 30).

Aquí se insinúa una pugna frontal entre razón y deseo por el contraste entre la opinión universal (propia de la razón) y el apetito. Señala claramente que la opinión (en este caso, 
conocimiento que no es universal) "no se opone a la razón por sí misma". Y añade: "es el deseo, no la opinión, el que es contrario a la recta razón” (Aristóteles, 1999, 1147 a 33).

Otro ejemplo lo encontramos un poco más adelante, también en el libro séptimo. Se refiere al incontinente y su contrario. En el continente hay un enfrentamiento entre razón y deseo, y es susceptible de ser persuadido, pero no cede: “...no cede por pasión o apetito ya que en ocasiones el continente será propicio a la persuasión; en cambio los otros es a la razón a la que no atienden, puesto que son susceptibles de apetitos y muchos de ellos son arrastrados por los placeres" (Aristóteles, 1999, 1151 b 15).

Este conflicto, tal como se presenta en el libro séptimo de EN se refiere a la polémica propia del continente y el incontinente. Las versiones intelectualistas se focalizan en estas sugerencias, ignorando otros textos en que la relación entre las diversas potencias del alma es más flexible y presenta esta tensión en términos diferentes. Lo interesante y nuevo es ver que los textos que apoyan el intelectualismo son los menos, y los que aplican la noción de deseo intelectivo superando esa constricción, son los más numerosos.

Si estos pasajes se toman como base de una teoría completa de la acción y de las facultades humanas, se ignoran otros textos de los que nos ocuparemos más adelante, y en los que se puede apreciar una mayor integración de conocimiento y deseo. Como consecuencia de este reduccionismo, se entiende la valoración moral exclusivamente dependiente de la razón y desvinculada de las facultades desiderativas. Además, se asume que la felicidad depende sólo del conocimiento, en este caso, en forma de juicio. También se la sitúa en la parte teórica del alma, lo cual obedece a los textos aristotélicos; pero desvinculada de la parte sensible, lo cual no responde a ningún pasaje de las Éticas. Esta es una comprensión "racionalista" de la felicidad aristotélica, que si bien puede desprenderse de algún pasaje concreto no responde al conjunto de la teoría antropológica y ética del estagirita. Desde esta concepción siempre se encuentra una tensión entre razón y apetito. Varios autores lo ven así. La explicación de Bostock puede servir de ejemplo:

Volumen 2. Número 1. Enero - Junio 2022 
Aristotle notes a certain, intense conflict in the soul between reason and desire, where one wants to go in one direction and the other in another direction. (He borrows this approach from Plato's Republic, 436b - 439d). However, while Plato thereby contends that there are three parts of the soul, Aristotle is content here with two. (...) His only basis for saying that the desiderative part 'participates in some aspect of reason' is that it can oppose and obey reason, and it can (in a virtuous man) coexist with it in harmony (1102 b 14-28). However, it seems that Aristotle mischaracterizes this when he continues to call it a desiderative part and, more generally, an appetitive part (epithumétikon, kai holós orektikon, 1102 b 30). (Bostock, 2000, p. 33)

En esta exposición han de advertirse dos problemas: primero, que el conflicto moral queda situado entre razón y apetito; siendo que el valor moral está en la razón y el deseo se le opone. Segundo, y como consecuencia de ello, únicamente la razón tiene legitimidad moral: ella es la que juzga rectamente, mientras que el apetito solo se alinea o discrepa respecto de ella. Es decir, el papel del deseo en la ética sería de mera obediencia a la razón. En sentido estricto sólo la razón tiene valor moral. Esta conclusión necesaria evidencia lo lejano que es el intelectualismo de la obra aristotélica en su conjunto y en su contexto. Cuando se focaliza todo el valor de la acción, y por tanto todo el valor moral, en la razón; entonces se cae en una doble confusión: entre los dos niveles de facultades, a saber, intelectivos y sensibles, y entre dos modos diversos de acción que son conocimiento y apetito.

García Valiño analiza el conflicto en términos platónicos refiriéndose a la doctrina platónica, afirma lo siguiente: "Platón observa que, en el alma humana, se produce a menudo un desacuerdo o conflicto entre la dimensión intelectiva y la sensitiva: entre nous y orexis". (García-Valiño, 2009, p. 85). La tensión entre facultades intelectivas y apetitivas se reduce a la tensión nous/orexis. En consecuencia, orexis se reduce al apetito sensible, pues se contrapone a las facultades intelectivas en general. Otra consecuencia es que la dimensión intelectiva sólo se compone de conocimiento y carece de facultades desiderativas o apetitivas. 
El mismo autor reconoce el problema a que esto da lugar, pues esta comprensión de las facultades del alma impide comprender la acción en el nivel intelectivo del alma, o cualquier movimiento suyo; pues el conocimiento solo no mueve nada (cfr. García-Valiño, 2009, p. 85). Esta observación se apoya, sobre todo, en dos textos fundamentales: la Ética Nicomaquea (1139 a 35) y el De Anima (433 a 15-24). Este aspecto, más relacionado con la acción, marca la diferencia entre Platón y Aristóteles y decide el planteamiento ético de Aristóteles.

Otro modo de expresar el mismo esquema es distinguir dos partes del alma con una actividad propia cada una: "una parte, intelectual, es la que piensa. La otra es la que desea." Whiting lo cuestiona, reconoce que Aristóteles no parece aceptar esta distinción claramente, pero considera que tampoco lo niega de modo concluyente:

Aristotle explicitly raises the question of whether one part of the soul thinks while another (appetitive) part wants. And he contends that the previous argument presented in (411 b 5; 402 b 9-13) supposedly rejects the seemingly basic distinction between a thinking party and a party that (apparently) desires (...) regarding võ $\varsigma$ and theoretical capacity, nothing is clear yet, but it seems to be a different kind of soul, and only this can be separated, like the eternal is separate from the perishable (De Anima $413 \mathrm{~b} 24-$ 32) ... distinguishing between theoretical capacity from those associated with locomotion and action - and therefore the capacities (or perhaps the parts of the soul in which they reside) responsible for perception, imagination and desire). (Whiting, 2002, pp. 148 y 150)

De nuevo, vemos cómo se confunde la parte teórica del alma con la parte cognoscitiva: como si toda ella consistiera únicamente en conocimiento. Por su parte, el nivel no intelectivo quedaría como completamente ajeno a la razón y sin una clara interacción con ella. Así se vería comprometido algo tan sencillo, en el marco de la filosofía aristotélica, como es el conocimiento sensible. Pero también queda cerrada la posibilidad de concebir un deseo intelectivo, un apetito vinculado a la razón. Todo deseo sería sólo y siempre sensitivo. Y el 
alma contemplativa tendría como rasgo esencial el conocimiento, aislado de toda potencia desiderativa.

Es lógico que Whiting, al inicio de su exhaustivo estudio sobre las partes del alma describa así sus potencias, porque se focaliza en el movimiento local. Sin embargo, esta interpretación de las partes del alma no se sostiene en el contexto de la teoría aristotélica de la actividad humana. Cuando examina el papel del nous, el propio Whiting encuentra algo que no encaja en sus ideas, puesto que la posibilidad de acción exige que haya interacción entre conocimiento y deseo. De nuevo, el problema que muestra que algo está equivocado en la interpretación asumida es la imposibilidad del movimiento (Cfr. Whiting, 2002, p. 182).

El conocimiento necesita del apetito para dar lugar a la acción. El apetito puede estar vinculado o no a la razón. Las facultades racionales no se reducen a conocimiento, sino que incluyen el querer de modo racional. Es necesario reconocer, por un lado, potencias cognoscitivas y desiderativas; $y$, por otro, ver que en ambos casos hay potencias vinculadas a la razón y otras que no lo están. Este análisis da lugar a cuatro tipos de potencias del alma: cognoscitivas racionales, cognoscitivas no racionales, apetitivas racionales y apetitivas no racionales. Por tanto, hay un tipo de deseo que es propiamente deseo, a la vez que está vinculado a la razón. Esto es el deseo intelectivo.

\section{Conflicto entre dos tipos de apetito}

Entendimiento y razón son cognitivos, mientras que la voluntad es apetitiva. Pero la voluntad, a la vez que apetitiva es racional porque está vinculada a la razón. La voluntad no es racional como una función propia de la razón, sino porque tiene un vínculo con ella. La interpretación intelectualista de la Ética aristotélica ha ignorado la presencia de facultades desiderativas entre las potencias intelectivas, que son todas las que tienen vínculo con la razón, las que conforman la parte de alma contemplativa. En la sección anterior hemos visto que las funciones intelectivas no consisten únicamente en el entendimiento, sino que se reconocen varias funciones y potencias que la componen. 
Comprender el concepto de deseo intelectivo mejora el análisis de los conflictos éticos. Las tensiones más fundamentales de la acción moral no se generan entre nous y orexis sino, como veremos a continuación, entre lo racional y lo no racional. El deseo racional por excelencia es la boulesis (querer) mientras que los típicos deseos no racionales son thymos (como pasión) y epithumía (como apetito) (Pearson, 2016, p.5).

La diferencia entre deseos racionales y no racionales obedece a la distinción que hace Aristóteles acerca de "una parte del alma que es irracional y otra que es racional" (Aristóteles, 1999,1102 a 25) (oion to men alogon autés einai, to delogon ejon), y esta parte irracional es "vegetativa y la cusa de la nutrición y el crecimiento" (Aristóteles, 1999, 1102 a 30). Segunda, hay otra parte del alma que es sólo en cierta medida racional; es irracional pero participa de algún modo de la razón (tes psijes alogos einai, metejousa mentoi pei logou) (Aristóteles, 1999, 1102 b 10). Esta segunda parte del alma no es ella misma racional porque sus funciones no están vinculadas directamente a la razón; pero parece que tienen una relación indirecta con ella. Se afirma de ella que puede ser persuadida (Aristóteles, 1999, 1102 b 32) por la razón de modo parecido a como un hijo escucha a su padre. Y otra parte del alma, en la que debe estar la función propia del ser humano es el alma intelectiva (Aristóteles, 1999, 1102 b 35).

Una vez que se asume esta comprensión de las diversas partes del alma, se podría preguntar en cuál de ellas se ubica el deseo. Pero la pregunta misma sería un error. Vimos al inicio que la existencia del deseo en un único modo sólo sensible no se sostiene. Entonces mostrábamos la necesidad de un deseo intelectivo. Ahora resulta todavía más claro: en todas las partes del alma hay deseo. En realidad, eso es coherente con la cuestión también repetida sobre el movimiento: cada conocimiento está vinculado a un tipo de deseo y su modo es diverso en cada parte del alma:

Añádase a esta la parte desiderativa, que parece distinguirse de todas tanto por su definición como por su potencia; sin embargo, sería absurdo separarla: en efecto, la volición se origina en la parte racional, así como el apetito y los impulsos se originan 
en la irracional; luego si el alma está constituida por estas tres partes, en cada una de ellas tendrá lugar el deseo. (Aristóteles, 2020, 432 b 5)

Este texto muestra la concordancia entre De Anima y Ética Nicomaquea. Tres tipos de deseo a los que ya hicimos referencia epithumia - un apetito o deseo corporal; thumos -pasión, emoción y otros tipos de impulsos; y boulesis un deseo racional o voluntad. Aristóteles señala diferencias entre las tres partes del alma y sus distintas relaciones con la razón.

Esta diversidad de modos de deseo es coherente con un principio esencial de la ética aristotélica: todo tiende a un bien. Esta relación de los tipos de deseo y distintos tipos de bienes, la vemos reflejada en la exposición de los distintos tipos de vida (Aristóteles, 1999, 1095 b 15-25) o la explicación de la función propia (Cfr. Aristóteles, 1999, 1098a). Se va clarificando el concepto de deseo intelectivo. Por ahora vemos que es un tipo de deseo que Aristóteles considera y explica, distinguiéndolo de otros modos de deseo. El deseo intelectivo está en la esfera intelectiva. Y, por tanto, las facultades propias de la contemplación incluyen una dimensión desiderativa.

Hay que tener presente que Aristóteles nunca señala desacuerdo entre las partes apetitivas y cognoscitivas, sino entre las racionales y las que no lo son. Por tanto, la verdadera confrontación no es tanto entre razón y deseo; aunque exista alguna alusión a ello; sino entre deseos racionales y no racionales. Así lo ha explicado Garrocho:

En el hombre continente la recta razón no se opone a la orexis sino que se enfrenta a la apetencia inmediata de la epithumia. Es decir, no es un enfrentamiento entre la razón y el deseo sino un conflicto interno a las propias determinaciones de la orexis. (...) La clave reside, por tanto, no en el enfrentamiento que se daría entre el deseo y la razón sino entre los distintos tipos de deseos entre los que se incluiría una forma específica que, como la boulesis, parece exigir el concurso de la razón. (Garrocho, 2017, p. 51) 
Hemos visto por tanto que no hay una contradicción necesaria nous-orexis o razón-deseo. No hay enfrentamiento entre ellos en cuanto tales, aunque pueda haber alguna situación en que se vean enfrentados. Como facultades en sí mismas, y vistas en el conjunto de la Ética; no son facultades en contradicción. Podemos matizarlo o aclararlo aún un poco más. Margot ha insistido en que no hay contradicción conceptual:

The eventual opposition of boulesis against orexis is not a logical contradiction since it is never the case that one wants both a thing and its inverse. Good is always the goal; however, we do not always know how to identify it or distinguish it from something that seems good. If desires can be reconciled, and if reason (logos) and desire (orexis) are contradictory, it is because in "animals that can perceive time" that the intellect's foresight does not always opt for immediate satisfaction. (Margot, 2008, p. 198)

La clave para descubrir la falsedad de algunas contradicciones que se han divulgado está en comprender el deseo en su sentido más amplio y más genérico (Margot, 2008, p. 196). Como hemos señalado desde el principio de esta sección, el deseo existe en formas diversas: como deseo intelectivo y como deseo no vinculado a la razón, que se plasma en thymos o epithymia.

La variedad de modos desiderativos juega un papel importante en la virtud. El virtuoso es aquél que consigue la armonía entre diversos tipos de deseo: son agradables las cosas conformes a la virtud para el que ama la virtud. El virtuoso se complace en las cosas nobles, encuentra placer en ellas, realiza las acciones que le hacen feliz... (Aristóteles, 1999, 1099 a $10)$.

La diversidad de deseo muestra que hay unidad, pero no unificación. Es decir, hay cierta unidad porque todos los modos de deseo, son tendencias apetitivas. Pero no hay unificación porque cada modo de deseo es distinto de los demás: el deseo puede ser racional o no. Y a la vez, cada tipo de deseo es un principio que dirige la acción y tiene sus propios matices. Por ejemplo, la boulesis no es siempre exactamente igual: es necesario "captar el funcionamiento de este híbrido, deseo típicamente aristotélico, la boulesis" (Monteils-Laeng, 2013, p. 442). 


\section{El deseo intelectivo como Boulesis}

Pearson afirma que "Boulesis es orexis del bien" (2016, p. 11). Son varios los autores que, de modo más bien indirecto, han tratado del deseo intelectivo en Aristóteles. Rorty, ha subrayado su papel en la contemplación (Rorty, 1980). Irwin señala la naturaleza racional del deseo del bien (Irwin, 1980). Mele resulta determinante pues analizando la relación del deseo intelectivo con otras facultades o actividades del alma (Mele, 1984). Lear ha destacado la relación de la finalidad con la eudaimonia, precisando algunos de sus aspectos y permitiendo una mejor comprensión de la identidad entre ambos factores dentro del ámbito intelectivo (Lear, 2004). Maher ha desarrollado la idea de amistad contemplativa, clarificando el modo en que la amistad tiene naturaleza racional (Maher, 2012).

Pero para comprender la importancia del deseo intelectivo es necesario distinguir boulesis y prohairesis, y reconocer que ambas son formas de este tipo de deseo. Boulesis se traduce normalmente como "querer", y se considera la palabra griega más cercana a "voluntad". "Hanna Arendt la considera el precursor conceptual de "voluntad" como la entendemos hoy (...) pero lo que nos interesa es definir "de qué tipo de deseo se trata y cómo se relaciona con el entendimiento" (García-Valiño, 2009, p.180).

En efecto, boulesis es el más claro y más generalmente reconocido deseo intelectivo. Los textos son específicos en este punto y el acuerdo unánime. Aristóteles afirma directamente en De Anima:

La observación demuestra que el intelecto no mueve sin deseo: la volición es, desde luego, un tipo de deseo y cuando uno se mueve en virtud del razonamiento es que se mueve en virtud de una volición (...) lo que causa el movimiento es siempre el objeto deseable que, a su vez, es lo bueno o lo que se presenta como bueno. Pero no cualquier objeto bueno, sino el bien realizable a través de la acción (...) es pues evidente que la potencia motriz del alma es lo que se llama deseo (orexis). (Aristóteles, 2020, 433a 23-30) 
Uno de los factores decisivos en la relación de razón y deseo es la distinta percepción de lo inmediato y el largo plazo. Sólo la razón puede comprender el bien a largo plazo y dirigirse a él. El deseo muchas veces se dirige a un bien inmediato porque es el que capta más claramente. El deseo corporal o apetito, típicamente busca el placer a corto plazo porque no tiene sentido del futuro (Cfr. Aristóteles, 2020, 433 b 5-10).

Hay dos elementos que definen el deseo intelectivo: que está ligado al bien y a la contemplación de esencias. Examinemos primero el deseo del bien. Toda actividad sigue a un deseo. Un deseo específico como el que estamos describiendo (el deseo intelectivo) genera sus propias acciones. La actividad del alma contemplativa sigue el deseo del bien. Hay una correspondencia entre deseo intelectivo y finalidad: "If we carefully study intellectual love we are carried to finality, and if we study finality responsibly we are brought to intellectual love. The reason for this is that finality in act is intellectual love in its characteristic of passion" (Rossner, 1972, p. 48).

El deseo intelectivo, que es propio del alma intelectiva, es el único que se dirige al bien como tal. Esto es porque el deseo de bien requiere el concepto de bien y la identificación de lo bueno como siendo bueno: "De los tres tipos de deseo, sólo la voluntad depende de tener el concepto de bien, y esto es algo que sólo tienen los seres racionales" (Mele, 1984, p.147). Los tres tipos de bien son conocidos perfectamente porque en el libro VIII de la Ética Nicomaquea, Aristóteles afirma que todo lo que puede ser amado (to fileton) es "bueno, placentero o útil" (Aristóteles, 1999, 1155 b 15). Los distintos tipos de deseo responden a diversos objetos, porque cada objeto conviene a diferentes facultades: el deseo físico es deseado sobre todo por el cuerpo y esas tendencias directamente afectadas por el placer. Las distintas pasiones tienen sus propias tendencias y se sienten en distintas partes del alma sensible. Presentes en el alma teórica están las facultades capaces de identificar el bien como tal, de pensar en él, y de decidir una acción que se dirige a lograrlo. En efecto, "lo que debe ser resultado de deliberación sobre todo bien de uno mismo. Aristóteles no quiere decir que no pueda distinguirse la voluntad de otros deseos, como de hecho querría" (Irwin, 1978, p. 257). La necesidad de deliberación establece que el deseo del alma contemplativa es un deseo racional. 
Tal como hemos descrito el deseo intelectivo en la sección anterior, es un querer vinculado al nous y que gobierna el comportamiento del hombre virtuoso. Esto es por lo que debe ser deseo del bien, y "un deseo de algo Bueno en la medida en que lo Bueno es un deseo racional" (Irwin, 1980, p. 45), porque se necesita el concepto de bien y la habilidad de guiarse por él.

"A menos que el bien atraiga a la persona el amor es imposible. Algo puede ser Bueno en realidad, y puedo saber que es atractivo y que atrae a algunas personas, sin embargo, puede que a mí me deje frío" (Rossner, 1972, p. 51). El amor es una atracción porque la pasión dentro de la voluntad es voluntaria (Rossner, 1972, p. 57). En otras palabras, el amor depende de dos principios, el bien y la voluntad. Cuando analizamos esa relación real de dependencia de la voluntad encontramos un objeto real, un término y un fundamento. El sujeto de la dependencia es, por supuesto, el amor y el término del que depende es ahora la voluntad como su causa eficiente próxima.

Esta es una tendencia distinta a la sensible en la que no se necesita ningún concepto, pero en la cual percepciones y sensaciones producen respuestas emocionales. De todos modos, un deseo del alma racional es distinto porque asume también la capacidad de bien de la motivación: "La explicación de este atributo sorprendente de que la parte racional del alma alberga deseo en ella, sólo puede ser que este deseo es necesario para tener motivación de obtener el bien humano" (Grönroos, 2015, p. 64).

El bien humano (que corresponde al ser humano en cuanto tal) exige entendimiento para ser deseado. Es la realización plena del ser humano, y el sujeto debe comprenderlo como tal para orientar su actividad en esa dirección. Es un bien que inspira acción cuando uno entiende lo que es Bueno, porque consiste en una acción que uno realiza. Y a la vez, exige compromiso a nivel del entendimiento. Sin embargo, esta actividad no está limitada al razonamiento o al entendimiento, sino a lo largo del deseo y del entendimiento hay un deseo y una motivación producida por y pasada en lo que uno conoce y entiende. 
El alma humana alberga un deseo por un bien intelectivo. Esta conclusión a la que hemos llegado estudiando la motivación y el movimiento, se revela también al analizar la función propia del ser humano. La función propia incluye un deseo orientado al bien en su nivel más alto. Esta actividad orientada al bien la define Aristóteles como ergon:

Decimos que la función del hombre es una cierta vida, y ésta una actividad del alma y acciones razonables, y la del hombre bueno estas mismas cosas bien $\mathrm{y}$ primorosamente, y cada una se realiza bien según la virtud adecuada; y, si esto es así, el bien humano es una actividad del alma conforme a la virtud, y si las virtudes son varias, conforme a la mejor y más perfecta, y además en una vida entera. (Aristóteles, 1999, 1098 a 14-16)

La idea de bien requiere que los objetos de contemplación sean amados además de conocidos. Son objetos de conocimiento y de amor en la medida en que hay una razón de bien en ellos, que exige racionalidad. Amor contemplativo es una elección y elección siempre comporta razonamiento y reflexión (Aristóteles, 1999, 1112 a 18). La elección (prohairesis) es un acto del alma contemplativa en el que nous y orexis actúan en acuerdo. Es un deseo vinculado a la razón, porque es la razón la que pone orden en los deseos (Cfr. Aristóteles, 1999, 1119 b 15). Sobre esto volveremos en la siguiente sección.

La contemplación se enfoca a lo esencial, lo eterno y lo inmutable, pero todo eso es también amado. Ciertamente, la contemplación es un fin en sí mismo: el conocimiento contemplativo es contemplar la verdad y no una aplicación práctica de algo verdadero. "Se ve así en el ejemplo del arquitecto y el geómetra: (el matemático) busca el ángulo recto, no por su aplicación sino por contemplar la verdad" (Aristóteles, 1999, 1098 a 30).

El objeto propio de la contemplación es, sin duda, lo inmóvil; pero eso no reduce los objetos de contemplación a las estrellas fijas o al motor inmóvil. Hay otras realidades móviles pero que tienen una esencia inmóvil que permanece, y que es propia de todas las esencias justo por ser esencias. Rorty ha señalado que la contemplación puede ser de las esencias de algo que es 
móvil: “Aunque el principal y paradigmático objeto de la contemplación es lo divino, las estrellas fijas, y quizás los objetos matemáticos, la condición para que algo sea contemplado es que sea necesario, inmutable, eterno, completo y noble (Aristóteles, 1999, 1139 a 6-8). Es también posible contemplar la forma inmutable de lo que cambia” (Rorty, 1980, p. 378).

Esta idea sitúa el problema del amor dentro de un marco distinto del habitual. Los seres humanos son a la vez sujetos y objetos de contemplación. Hemos visto que hay un tipo de amor que está incluido en la actividad contemplativa. Ahora podemos añadir que es un amor de lo esencial. Esto tiene una consecuencia muy interesante en relación al amor entre las personas: es posible querer más allá de lo accidental: Aristóteles detalla todo esto en el libro octavo de la Ética Nicomaquea. Su propuesta permite enfatizar la posibilidad de contemplación entre las personas porque "podemos contemplar la actividad de la vida moral en el mismo modo que contemplamos el cielo estrellado en lo alto" (Rorty, 1980, p. 378). Y esta es una doble contemplación: contemplamos - conociendo y amando a la vez- la vida moral que es la actividad propia del alma contemplativa del sujeto.

Empezábamos esta sección afirmando que el amor del bien es racional en la medida en que es del bien. Hemos visto también que la racionalidad del bien es similar a la de las esencias o formas, que identifica el bien en cada cosa a la que se dirige, trascendiendo idiosincrasias y reconociendo el bien en sus diversas manifestaciones. Uno de los conceptos más claros en esta dirección y reconocimiento es la finalidad.

\section{Prohairesis como un tipo de orexis. Unión de orexis y nous}

El análisis de la boulesis nos ha llevado a la elección (prohairesis) para analizar la cuestión de cómo cooperan nous y orexis. Nos interesaba estudiar la dimensión propiamente contemplativa del deseo intelectivo, partiendo de la boulesis. Pero ahora podemos volver de nuevo a esta cuestión sobre el modo peculiar de deseo que es prohairesis y su también especial unión con el nous. 
Son escasos los estudios de prohairesis como orexis (Vigo, 2012, p. 3). Sorabji la menciona como un tipo de deseo, la causa eficiente de la praxis, como acción deliberada y es un tipo de deseo que se orienta a la elección de los medios para un fin (Sorabji, 1974, p. 81). Como señala A. Vigo Kullmann (1945) es uno de los pocos que estudia directamente las razones por las que considerarla un tipo de deseo. Esos motivos se peuden resumir en tres: 1) porque es deseo voluntario 2) porque se distingue de thymos y epithumia y 3) porque se distingue de boulesis (Kullmann, 1945, pp. 111-113).

Aunque esté intrínsecamente ligado al conocimiento, no es fácil definir claramente si es una facultad del apetito o del intelecto. Parece que posee partes iguales de ambos. Quizás por este motivo algunos autores la han confundido con la phronesis. Prohairesis no es una facultad del razonamiento práctico, como afirma, por ejemplo, García-Valiño sugiere que "podemos interpretar prohairesis como una facultad que pertenece exclusivamente a agentes racionales: el poder de escoger y decidir, tomar una elección deliberada de preferencia. Es una facultad de razón práctica: de dianoia o phronesis".

En la elección entendimiento y deseo actúan conjuntamente. Aristóteles afirma que "La elección (prohairesis) es un deseo deliberado (orexis bouletiké)" (Aristóteles, 1999, 1139a 25) es "inteligencia deseosa (orektikós nous) o deseo inteligente (orexis dianoetiké)" (Aristóteles, 1999,1139 b 5. Este es probablemente el pasaje más representativo para la comprensión del modo en que se unen orexis y nous. No sólo no están necesariamente en pugna, como habíamos comentado anteriormente. No solo son compatibles. Son necesarios uno al otro para que se dé la elección. La elección consiste precisamente en una unión de ambos tan equitativa, que no se puede priorizar a uno sobre el otro. La expresión "inteligencia deseosa o deseo inteligente" indica esta compenetración y equidad.

Mysen ofrece una breve explicación sobre la interacción de ambas potencias:

La causa de la decisión es el deseo. El deseo a su vez es "informado" por la deliberación (1139b4-5). La función de la deliberación es conseguir una ruta directa a 
la acción por "retornar el fin a nosotros" (1113a5-6). La decisión (prohairesis) es una combinación de pensamiento (dianoia), inteligencia (nous), y deseo (orexis). Después de la deliberación una decisión nos llevará a realizar lo que la buena deliberación prescribe. (Mysen, 2011, p. 68)

“Orexis y nous comparten una función que es mostrar lo deseable. El objeto de deseo, además de ser conocido se reconoce como deseable" (Monteils-Laeng, 2013, p.454). Es necesario tener noticia (a través de alguna facultad cognoscitiva) del objeto y tener una representación que incluya o permita valoraciones. También es necesaria una valoración (que es de alguna facultad apetitiva) que mueva a la acción que nos acerca al objeto deseado.

También es interesante prestar atención a la presencia de conceptos en el acto desiderativo. Porque el deseo es la percepción de lo bueno y malo en cuanto tal (Tuozzo, 1994, p. 536) y puede ser conceptualizado o no: cuando está conceptualizado incluye pensamiento, cuando no está conceptualizado incluye solo percepción. (Tuozzo, 1994, p. 542).

...el objeto de deliberación es algo que está en nuestro poder y es tema de deliberación y deseable, la elección será también un deseo deliberado de cosas a nuestro alcance; porque cuando decidimos después de deliberar, deseamos de acuerdo con la deliberación. (Aristóteles, 1999, 1113 a 10)

¿Podemos concluir que prohairesis es un tipo de orexis? En primer lugar y desde el punto de vista etimológico, hay razones para considerarlo así: el componente del sustantivo "aireo" significa elegir o escoger. Tiene una connotación de deseo que es quizás más clara en otra palabra de la misma raíz: airetos, que significa elegible o deseable. Airesis es tomar algo que se prefiere. Es un modo de querer, por lo que prohairesis es un modo de volición que no puede ignorarse cuando se estudia el deseo en Aristóteles y se quiere analizar en todas sus formas.

El libro tercero de Ética Nicomaquea prohairesis es definida como una forma específica de lo voluntario (ecousiou): 
"La elección (prohairesis) es manifiestamente algo voluntario, pero no se identifica con lo voluntario, que tiene más extensión: de lo voluntario participan también los niños y los otros animales, pero no de la elección, y a las acciones súbitas las llamamos voluntarias, pero no elegidas". EN III, 2, 1111 b 4.

Esta observación sitúa claramente la elección como una potencia desiderativa y no cognoscitiva.

Stoyles hace referencia a las observaciones de Kenny (1979) y Irwin (1992): Aunque discuten su sentido y desaprueban su asimilación a la idea moderna de voluntad vieron que esta interpretación era ajustada: entender prohairesis en un sentido fuerte de voluntad, como muchos académicos, hace esta lectura de Aristóteles especialmente tentadora, porque deja espacio a la teoría de Aristóteles que justifica algo parecido a lo que nosotros decimos al afirmar que la voluntad puede superar deseos inferiores (Stoyles, 2007, p. 201).

En efecto, uno de los sentidos de prohairesis es el de máximo tipo de deseo, con el sentido específico de que es el modo desiderativo más cercano a la razón. Sería el tipo más alto en una jerarquía donde lo racional es superior y lo corporal es inferior. Esta jerarquía estaría establecida por los tres niveles de funciones vitales: vegetativa, sensitiva y racional. Prohairesis es un deseo racional.

\section{Conclusiones}

Las facultades intelectivas, según Aristóteles, incluyen el deseo. Hay al menos dos formas de deseo que Aristóteles considera racionales: boulesis y prohairesis. Son, propiamente, deseos, y están, a la vez, vinculados a la razón.

Las lecturas reduccionistas que han identificado la felicidad con una contemplación sólo cognoscitiva, o que han reducido la virtud a la actividad racional; tienen que ser 
reconsideradas ante formas de deseo que innegablemente forman parte de la contemplación, la felicidad y la virtud.

Si estos tres conceptos clave: contemplación, felicidad y virtud no son sólo razón, sino que como hemos mostrado en nuestro trabajo- incluyen el deseo; entonces el supuesto intelectualismo de Aristóteles quedaría claramente contradicho. Ya no es sólo la razón el elemento definitivo para fundamentar la ética, sino toda la parte teórica del alma, incluido el deseo presente en ella.

\section{Referencias}

Akrill, J. L. (1980). Aristotle on Eudaimonia, A. Rorty (Ed.), Essays on Aristotle's Ethics (pp. 15-33). University of California Press.

Aristóteles. (1999). Ética a Nicómaco. María Araujo y Julián Marías (Trads.). Centro de Estudios Políticos y Constitucionales.

Aristóteles, (2020). Acerca del alma (T. Calvo Trad.). Gredos.

Bostock, D. (2000). Aristotle's Ethics. Oxford University Press.

García-Valiño, J. (2009). Deseo racional y elección deliberada. los conceptos de boulesis y proairesis como precursores de la noción de voluntad. Universidad de Málaga. https://dadun.unav.edu/bitstream/10171/35529/1/CAF 212

Garrocho, D. (2017) El bien como objeto perceptivo de las pasiones en la epistemología moral de Aristóteles, Pensamiento, 73 (275), 45-60. DOI:10.14422/pen. v73.i275.y2017.003

Grönroos, G. (2015). Wish, Motivation and the Human Good in Aristotle, Phronesis, 60, 6087. 
Irwin, T. (1978). First principles in Aristotle's Ethics, P. A. French, T. E. Uehling, and H. K. Wettstein, (Eds.), Midwest Studies in Philosophy (pp. 252-272). University of Minnesota Press.

Irwin, T. (1980). The Metaphysical and Psychological Basis of Aristotle's Ethics, A. Rorty (Ed.), Essays on Aristotle’s Ethics (pp. 35 -53). University of California Press.

Kraut, R. (1975). The importance of love in Aristotle's ethics. Philosophy Research Archives, $1,300-322$.

Kullmann, E. (1945). Beiträge zum aristotelischen begriff der “Prohairesis". Dissertation zur erlangung der doktorwürde der Hohen Philosophish-Historischen Fakultät der Universität Basel.

Lear, G. R. (2004). Happy Lives and the Highest Good: An Essay on Aristotle's Nicomachean Ethics, Princeton University.

Maher, D. (2012). Contemplative Friendship in Nicomachean Ethics, Review of Metaphysics, 65 (4), pp. 765-794.

Margot, J.P. (2008). Aristóteles: deseo y acción moral, Praxis Filosófica, 26, 189-202.

Mele, A. R. (1984) Aristotle's Wish, Journal of the History of Philosophy, 22, 139-156.

Monteils-Laeng, L. (2013). Aristote et l'invention du désir, Archives de Philosophie, 3 (76), 441-457.

Mysen, R. (2011). Desiring the Good: Motivational Development in Aristotle's Nicomachean Ethics. University of Bergen. 
Nagel, Th. (1980). “Aristotle on Eudaimonia”, A. Rorty (Ed.), Essays on Aristotle's Ethics, (pp. 7-14). University of California Press.

Pearson, G. (2016) Aristotle on Desire, Cambridge University Press.

Rorty, A. (1980). The place of contemplation in Aristotle's Nicomachean Ethics, Rorty, A. (Ed.), Essays on Aristotle’s Ethics, (pp. 377-394) University of California Press.

Rossner, W. (1972). The Process of Human Intellectual Love, or Spirating a Pondus, The Thomist: A Speculative Quarterly Review, 36 (1), pp. 39-74.

Sorabji, R. (1974). Body and Soul in Aristotle. Philosophy, 49 (187), pp. 63-89.

Stoyles, B. J. (2007) Aristotle, akrasia, and the place of desire in moral reasoning Ethical Theory and Moral Practice. (10) 195-207.

Tuozzo, Th. M. (1994). Conceptualized and Unconceptualized Desire in Aristotle, Journal of the History of Philosophy 3 (4)

Vigo, A. (2012). Deliberación y decisión según Aristóteles, Tópicos (43) 51-92.

Whiting, J. E. (2002) Locomotive soul: The Parts of Soul in Aristotle's Scientific Works, Oxford studies in Ancient Philosophy, (22) 141-200. 